\title{
Association of DnaK and GroEL with Antimicrobial Resistance in Salmonella Abortusovis
}

\author{
Luminita Monica Vanghele ${ }^{1}$, Maria Ionescu ${ }^{2}$, Handan Coste $^{3}$ and Elena Ganea ${ }^{4}$ \\ 1,2,3 Institute for Diagnosis and Animal Health, Bucharest, Romania \\ ${ }^{4}$ Institute of Biochemistry of the Romanian Academy, Bucharest, Romania
}

Correspondence should be addressed to: Luminita Monica Vanghele; vanghele.monica@idah.ro

Received date: 4 august 2014; Accepted date: 19 october 2014; Published date: 8 September 2016

Academic Editor: Marisa Ribeiro De Itapema Cardoso

Copyright (C) 2016. Luminita Monica Vanghele, Maria Ionescu, Handan Coste and Elena Ganea. Distributed under Creative Commons CC-BY 4.0

\begin{abstract}
Multidrug-resistant (MDR) strains of Salmonella are now encountered frequently and the rates of multidrug-resistance have increased considerably in recent years. The association of DnaK and GroEL molecular chaperones with the stress response induced by antibiotics has been mentioned for the most frequently isolated serovar, S. enterica subsp. enterica Typhimurium, but similar studies on $S$. Abortusovis were not published, so far. In the present work, the induction of DnaK and GroEL in $S$. Abortusovis cells under stress conditions caused by heat shock and by various antibiotics respectively was studied by Western blot and real time RT-PCR methods. The transcription and translation analyses showed increased levels of DnaK and GroEL after a temperature shift from $37^{\circ} \mathrm{C}$ to $42^{\circ} \mathrm{C}$, and after the exposure of a multiresistant $S$. Abortusovis strain to subinhibitory concentrations of various antimicrobials (nalidixic acid, streptomycin, kanamycin, tetracycline and gentamicin). The data presented here may provide a starting point for future research regarding the role of $S$. Abortusovis molecular chaperones in the stress response induced by antibiotics.
\end{abstract}

Keywords: Gene expression · Salmonella Abortusovis · molecular chaperones· antimicrobial resistance

\section{Introduction}

Salmonella enterica subsp. enterica serovar Abortusovis, a sheep-specific serovar, is the main cause of ovine abortions, stillbirths, and illness in lambs infected at birth in ovine flocks in some countries of Europe and also in Western Asia (Jack, 1968; Pardon et al, 1988; Uzzau et al., 2000). Sometimes it is difficult to make a treatment with antibiotics during an outbreak because the rate of multidrug-

Cite this Article as: Luminita Monica Vanghele, Maria Ionescu, Handan Coste and Elena Ganea (2016), "Association of DnaK and GroEL with Antimicrobial Resistance in Salmonella Abortusovis ", International Inurnal of Veterinarv Medicine: Research \& Renorts. Vol. 2016 (2016). Article ID 623135. 
resistance has increased considerably in recent years (Pardon et al., 1988). This may be a result of selective pressure, such as the intensive use of antibiotics for therapeutic needs but also for growth promotion (prophylactic) in farm animals. Many of the antimicrobials used for the animal diseases are identical or related to those used to treat infections among humans and the antimicrobial resistance of bacteria in animals (as in humans) may arise rapidly due to inappropriate use of antibiotics.

A stress response is a mechanism which protects bacterial polypeptides from misfolding and aggregation in the highly crowded cellular environment (Gomes and Simão, 2009). In bacteria, the major molecular chaperones, DnaK/DnaJ and GroE (GroEL and GroES) are involved in the quality control of the cells. Several reports have shown that GroEL/GroES and DnaK/DnaJ are induced during various environmental conditions (heat shock, acidic $\mathrm{pH}$, oxidative stress, osmotic and saline stress, UV radiation, ethanol, antibiotics, heavy metals, and aromatic compounds) in some pathogenic bacteria (e.g. Salmonella enterica subsp. enterica serovar Typhimurium, E. coli, Brucella, Lactococcus lactis, Caulobacter crescentus, Acinetobacter baumannii, A. calcoaceticus) (Morgan, 1986; Blaszczak et al., 1995; Bianchi and Baneyx, 1999; Benndorf et al., 1999; Benndorf et al., 2001; Prasad et al., 2003; Susin et al., 2006; Vanghele et al., 2013).
However, the importance of these chaperones for stress response in Salmonella enterica subsp. enterica serovar Abortusovis has not been comprehensively studied. In this study, the expression of DnaK and GroEL in multiresistant Salmonella Abortusovis, after exposure to heat shock and different antibiotics, was estimated by Western blot and quantitative real time RT-PCR and the results have been compared with those of bacteria grown under normal conditions.

\section{Materials and methods}

Bacterial strains and growth conditions; determination of Minimum Inhibitory Concentrations (MICs)

A multi-resistant strain $S$. Abortusovis 205 (Table 1) obtained from the Bacteriology Department, IDAH (Institute for Diagnosis and Animal Health), Romania, was used in this study. This strain was confirmed as Salmonella Abortusovis by standard serotyping procedures in accordance with the Kauffmann-White scheme (Grimont \& Weill, 2007) and PCR amplification of serovar-specific IS200 element (Beuzón C. R. et al., 1997). Salmonella cells were grown in buffered peptone water (BPW) under aerobic conditions for $21 \mathrm{~h}$ at $37^{\circ} \mathrm{C}$. The determination of Minimum Inhibitory Concentration (MIC) was done by Broth Microdilution assay according to CLSI guidelines (Clinical and Laboratory Standards Institute, 2005).

Table 1: Antimicrobial susceptibility of S. Abortusovis 205

\begin{tabular}{|l|c|}
\hline \multicolumn{1}{|c|}{ Antimicrobials } & MIC $(\mu \mathrm{g} / \mathbf{m l})$ \\
\hline & \\
Nalidixic acid (NA) & 64 \\
Streptomycin (S) & 32 \\
Kanamycin (K) & 8 \\
Tetracyclin (TE) & 64 \\
Gentamicin (CN) & 0.5 \\
& \\
\hline
\end{tabular}

The antimicrobial agents tested were purchased by Oxoid (UK) and were the following: nalidixic acid, gentamicin, kanamycin, streptomycin and tetracycline.
In parallel with the untreated culture (control culture) mentioned above, the stress conditions were induced by exposure bacterial cells to heat shock $\left(37^{\circ} \mathrm{C}\right.$ 
to $42^{\circ} \mathrm{C}$ temperature shift for $15 \mathrm{~min}$ ) and subinhibitory concentrations of various antibiotics (nalidixic acid $45 \mu \mathrm{g} \mathrm{ml} \mathrm{m}^{-1}$, gentamicin $0.37 \mu \mathrm{g} \mathrm{ml}-1$, kanamycin $6 \mu \mathrm{g}$ $\mathrm{ml}^{-1}$, streptomycin $22 \mu \mathrm{g} \mathrm{ml} \mathrm{ml}^{-1}$ and tetracycline $45 \mu \mathrm{g} \mathrm{ml}^{-1}$ ) for $30 \mathrm{~min}$ at $37^{\circ} \mathrm{C}$.

\section{Western blot analysis (immunoblot)}

Proteins were extracted with Bio Rad Ready Prep Protein Extraction kit and the identical protein concentration, as well as Broad Range molecular weight standard (Bio-Rad) were applied on polyacrylamide (PAA) gel. After electrophoresis, proteins were transferred to a PVDF (polyvinylidene difluoride) membrane using Semi-Dry Transfer Cell (BioRad) according to the instructions recommended by the manufacturer. In parallel, the gels were stained with $0.1 \%$ Coomassie Brilliant Blue R-250 for the control of electrophoresis. We used a solution containing methanol, acetic acid and water (4:1:5) to destain SDS-PAGE gels. The mouse monoclonal antibodies anti-GroEL and anti-DnaK were purchased from Stress Gen Biotechnologies Corporation. Detection of stress proteins was done with Immun-Blot Assay kit (BioRad).

\section{Survival assays}

S. Abortusovis 205 was grown in BPW for $21 \mathrm{~h}$ at $37^{\circ} \mathrm{C}$ and aliquots of culture were pretreated for $30 \mathrm{~min}$ with heat shock at $39^{\circ} \mathrm{C}$ and then exposed to stress caused by a subinhibitory concentration of $45 \mu \mathrm{g}$ nalidixic acid $\mathrm{ml}^{-1}$. The cultures were diluted after treatment and plated on Mueller Hinton agar to determine the number of viable bacterial cells.

\section{RNA extraction and reverse transcription (RT)}

RNA was stabilized in RNAprotect Bacteria Reagent (Qiagen). Extraction of total RNA from the bacterial cultures was performed with the Qiagen RNeasy Mini Protocol. The samples were treated with DNase I solution (RNase-Free DNase Set, Qiagen) and the absence of residual DNA contamination was confirmed by PCR. The quantity and purity of the total RNA were measured with a Bio Photometer Eppendorf. Complementary DNA (cDNA) was generated from total RNA with M-MLV RT according to the manufacturer's instructions (Promega) using $2 \mu \mathrm{M}$ of each reverse primer and $100 \mathrm{ng}$ total RNA (50 $\mu \mathrm{l}$ final volume).

\section{Controls for semi-quantitative and quantitative RT-PCR}

S. Abortusovis 205 grown in BPW in normal conditions was used as positive control. No-template controls were used for in vitro experiments as negative controls. A housekeeping gene (16S rRNA gene) was chosen as internal control for the normalization of cDNA samples in semi-quantitative RT-PCR analysis. This gene was used as a reference in real time quantitative RT-PCR, because its expression is relatively constant in bacteria.

\section{Real time quantitative PCR (qPCR) and semi-quantitative PCR}

Transcript detection analysis was carried out by real time quantitative PCR (qPCR) with HotStarTaq DNA Polymerase (Qiagen). Selected genes were amplified from normalized cDNA samples $(5 \mu \mathrm{l})$ with specific primers $(0.8 \mu \mathrm{M}$ of each primer) and probes $(0.5 \mu \mathrm{M}$ each probe) for Salmonella dnaK gene (accession no. U58360.1) and groEL gene (accession no. 1255856) were designed in this study with Primer3 software v.0.4.0: dnaK-Salm-FP 5'accggtaactgaagccgtta3'; dnaK-Salm-RP 5'cgccatcaacttcgtcgatt3'; dnaK-Salm-P 5'FAM-gctggcttacggtctggata-TAMRA3';

groEL-Salm-FP 5'tgcaggatatcgctacctg3'; groEL-Salm-RP 5'ctggatggcagcttcttcac3'; groEL-Salm-P 5'FAMacaccaccaccatcatcgat3'. The specific primers and probe for 16S rRNA gene (accession no.L26168) were designed by Vanghele (2013). For the semi-quantitative PCR, the amplicons were analysed on $1.5 \%$ agarose gel and visualized by ethidium bromide staining. Amplification program (iQ5 Real Time PCR System-BioRad): 15 min at $98^{\circ} \mathrm{C}$, then 50 cycles of $35 \mathrm{~s}$ at $95^{\circ}$, $45 \mathrm{~s}$ at $56^{\circ} \mathrm{C}$ and $30 \mathrm{~s}$ at $70^{\circ} \mathrm{C}$. The final extension was performed for $5 \mathrm{~min}$ at $70^{\circ} \mathrm{C}$. 
Relative transcriptional level was determined by the method of $2^{-\Delta \Delta_{\mathrm{Ct}}}$ (Livak and Schmittgen, 2001).

\section{Statistics}

Three replicates were used for each experiment. The relative transcription of the genes and the quantification of the proteins were calculated as the mean \pm the standard deviation (S.D.)

\section{Results and discussion}

The rapid emergence of Salmonella strains that are resistant to commonly used antimicrobials should be particularly noted by clinicians, microbiologists and those responsible for the control of communicable diseases, as well as the food producers and farmers. Control of drugresistant Salmonella is most efficiently achieved through the reduction of antimicrobial use, but another solution could be the development of novel antimicrobials. There is evidence that the bacterial molecular chaperones may be interesting therapeutic targets for novel molecules able to kill the resistant strains of Salmonella (Cardoso et al., 2010). For instance, pyrrhocoricin is able to kill tetracycline- and aminoglycoside-resistant strains of E. coli, Salmonella Typhimurium, Klebsiella pneumoniae, Haemophilus influenzae and Moraxella catarrhalis (Cudic et al., 2002).

In order to understand the role of molecular chaperones in antibiotic tolerance, the induction of DnaK and GroEL in S. Abortusovis cells under stress caused by heat shock and by various antibiotics was analysed at the transcriptional and translational levels and compared with bacteria grown under the normal conditions.
Previous studies on various bacteria, such as Escherichia coli, Salmonella Typhimurium, Staphylococcus aureus, Klebsiella pneumonia, Haemophilus influenza, Moraxella catarrhalis, except Salmonella Abortusovis, demonstrated the induction of the molecular chaperones under stress caused by antibiotics (Kragol et al., 2001; Singh et al., 2001; Yamaguchi et al., 2003; Cardoso et al., 2010). Cardoso et al. (2010) showed that molecular chaperones are upregulated at the transcriptional and translational levels by various antibiotics, which could increase the ability of bacterial cells to cope with the cell demage caused by antibiotics with other antibiotic-resistance machinery to maintain cell survival.

In order to analyse the role of molecular chaperones during the antibiotic response in S. Abortusovis, DnaK and GroEL expression was determined in bacterial cells incubated with subinhibitory concentrations of various antibiotics, also during heat shock. The expression of DnaK and GroEL was analysed at the transcriptional and translational levels by real time RT-PCR and Western blot using a multiresistant strain of S. Abortusovis, 205.

To estimate the translation level of DnaK and GroEL molecular chaperones, a Western blot assay was carried out for each selected gene. The levels of DnaK/GroEL protein expression for bacterial cells in normal conditions were used as positive controls. DnaK and GroEL proteins were identified by Western blot. The proteins cross-reacted with the specific antibodies with the strong reactions as shown in Figure 1 and Figure 2. The quantification of the bands intensity for DnaK and GroEL proteins from Western blots was done with UN-SCAN-IT gel $^{\mathrm{TM}}$ software. 


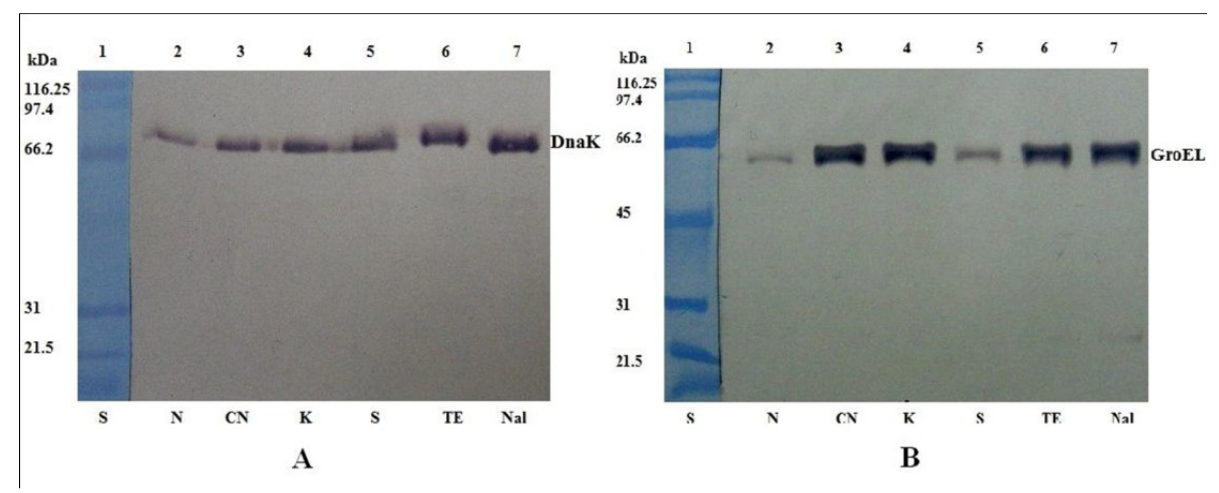

Figure 1: Western blot analysis of DnaK (A) and GroEL (B) expression in S. Abortusovis cells after exposure to various antibiotics

Lane 1: SDS-PAGE Molecular Weight Standard (kDa) (S); lane 2: normal conditions $(\mathrm{N})$; lane 3: gentamicin $(\mathrm{CN})$; lane 4: kanamycin $(\mathrm{K})$; lane 5: streptomycin
(S); lane 6: tetracycline (TE); lane 7: nalidixic acid (NA).

The translation levels of DnaK and GroEL proteins were increased after exposure to heat shock at $42^{\circ} \mathrm{C}$ (Figure 2).

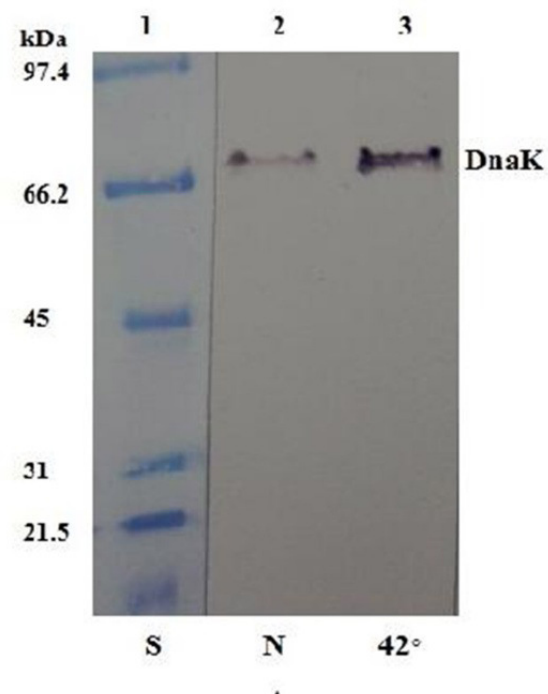

A

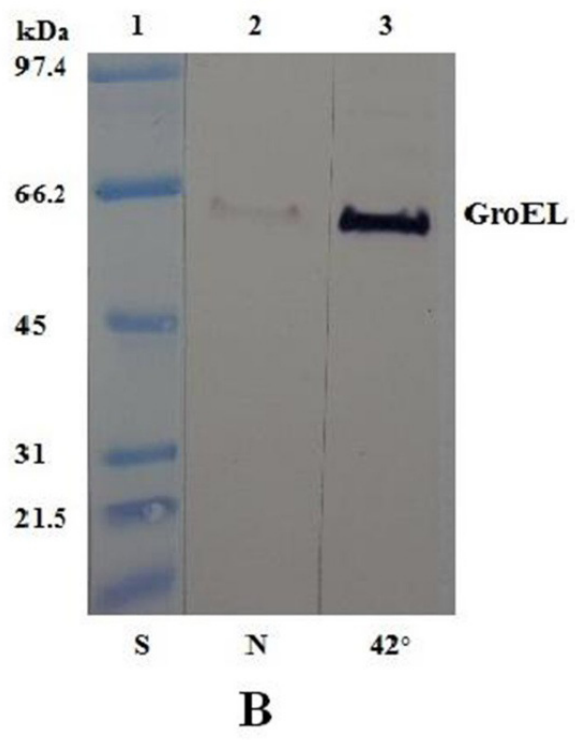

Figure 2: Western blot analysis of DnaK (A) and GroEL (B) expression in S. Abortusovis cells after heat shock.

Lane 1: SDS-PAGE Molecular Weight Standard (kDa) (S); lane 2: normal conditions $(\mathrm{N})$; lane 3 : heat shock $\left(42^{\circ}\right)$.

The protein levels corresponding to DnaK as a response of $\mathrm{S}$. Abortusovis to gentamicin, streptomycin and kanamycin are different depending on antibiotic (Figure 3). DnaK protein amount was increased after the incubation with nalidixic acid and tetracycline, approximately 4-fold higher than those observed in control culture (without antibiotic). 


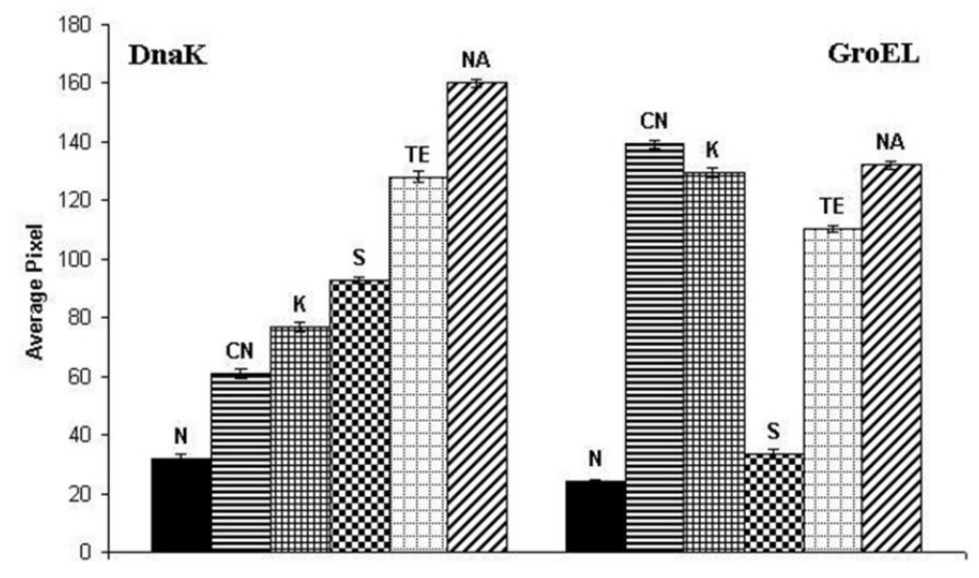

Figure 3: DnaK and GroEL proteins quantification by densitometric scanning of the membranes shown in Fig. 1 (average pixel)

Different antibiotics induce the expression of the DnaK and GroEL proteins in $\mathrm{S}$. Abortusovis: (N) normal conditions, (CN) gentamicin, (K) kanamycin, (S) streptomycin, (TE) tetracycline, (NA) nalidixic acid.

GroEL protein is up-regulated when S. Abortusovis was exposed to all antimicrobial drugs. There is a similar pattern of GroEL and DnaK protein expression in the presence of nalidixic acid and tetracycline. The GroEL protein level increased drastically after the exposure to the aminoglycoside antibiotics (gentamicin and kanamycin) (about 6-fold) (Figure 3). In contrast, the level of GroEL after the exposure to another aminoglycoside (streptomycin) was increased 1.5 -fold as compared to DnaK protein level. The low level of GroEL expression after exposure to streptomycin as compared with other aminoglycosides suggests that there is not a common mechanism for a certain group of antibiotics, because the involvement of the two molecular chaperones in antibiotic resistance is different.

A sensitive method for quantification of the gene expression, quantitative real time RTPCR, was performed in parallel with Western blot experiments to determine the transcription levels of the $d n a K$ and groEL. The $d n a K$ and groEL genes were amplified from normalized cDNA samples. The relative transcription (R) was calculated by using three replicates for each real time qRT-PCR experiment (Table 2). 
Table 2: Transcription of $d n a K$ and groEL measured by real time qRT-PCR in $S$. Abortusovis in stress conditions caused by heat shock and various antibiotics: $N$ : normal conditions; CN: gentamicin; K: kanamycin; S: streptomycin; TE: tetracycline; NA: nalidixic acid; R: relative transcription determined by the method of $2^{-\Delta \Delta_{C t}}$ (Livak and Schmittgen, 2001) \pm standard deviation (S.D.) of three replicates

\begin{tabular}{|l|l|l|l|l|l|c|c|}
\hline- & \multicolumn{9}{|c|}{ Relative transcription (R) } \\
\cline { 2 - 8 } & $\begin{array}{c}\text { Normal } \\
\text { condition } \\
\mathbf{s}(\mathbf{N})\end{array}$ & Heat $\mathbf{( 4 2}^{\circ} \mathbf{)}$ & $\begin{array}{c}\text { Gentamicin } \\
\mathbf{( C N )}\end{array}$ & $\begin{array}{c}\text { Kanamycin } \\
\mathbf{( K )}\end{array}$ & $\begin{array}{c}\text { Streptomycin } \\
\mathbf{( S )}\end{array}$ & $\begin{array}{c}\text { Tetracyclin } \\
\mathbf{e} \\
\text { (TE) }\end{array}$ & $\begin{array}{c}\text { Nalidixic acid } \\
\text { (NA) }\end{array}$ \\
\hline dnaK & $1 \pm 0.52$ & $2.32 \pm 0.46$ & $1.7 \pm 0.04$ & $2.24 \pm 0.07$ & $2.6 \pm 0.16$ & $4.1 \pm 0.62$ & $4.9 \pm 0.65$ \\
\hline groEL & $1 \pm 0.24$ & $7.4 \pm 0.8$ & $6.1 \pm 0.33$ & $5.9 \pm 0.09$ & $1.6 \pm 0.14$ & $4.4 \pm 0.16$ & $5.2 \pm 0.22$ \\
\hline
\end{tabular}

Although RNA dnaK presented a small difference between the expression levels observed after exposure to various antibiotics, the DnaK chaperone was highly induced by the three aminoglycoside antibiotics (gentamicin, streptomycin and kanamycin). The results of real time qRTPCR of dnaK gene showed an increase of transcription approximately 1.5 -fold after $30 \mathrm{~min}$ in the presence of gentamicin. The RNA dnaK level, as a response of $\mathrm{S}$. Abortusovis to streptomycin and kanamycin is different depending on antibiotic (2.5-fold and, respectively 2 -fold higher than those observed in control culture). The level of the dnaK transcript for nalidixic acid and tetracycline was approximately the same with the DnaK protein, also for the temperature shift at $42^{\circ} \mathrm{C}$ (Table 2). In contrast with the Western blot analysis, the levels of induction of groEL gene presented small variations depending on the antibiotic (Table 2).

The results for DnaK and GroEL molecular chaperones are confirmed by real time qRT-PCR method that showed a major increase in the level of dnaK and groEL transcripts in all experiments with antibiotics. These results suggest that the DnaK and GroEL molecular chaperones levels depend on genes transcription.

In order to evaluate the sensitivity of $\mathrm{S}$. Abortusovis to the antibiotic stress, the bacterial cells were pretreated for $30 \mathrm{~min}$ at $39^{\circ} \mathrm{C}$ and then exposed to a subinhibitory concentration of nalidixic acid. This pre-incubation induced an increased ability of bacteria to survive to nalidixic acid exposure than bacterial cells grown at physiological temperature $\left(37^{\circ} \mathrm{C}\right)$. Almost $90 \%$ of cells pretreated at $39^{\circ} \mathrm{C}$ survived to antibiotic exposure comparative with cells incubated at $37^{\circ} \mathrm{C}$ (55\% relative survival) (Figure 4 ). The good survival of bacterial cells to high temperature and antibiotic exposure and the drastic increase of the molecular chaperones expression after pretreatment at the moderate temperature were mentioned previously for Salmonella Typhimurium (Mackey and Derreck, 1990) and Acinetobacter baumannii (Cardoso et al., 2010), but never described in $S$. Abortusovis, so far. 


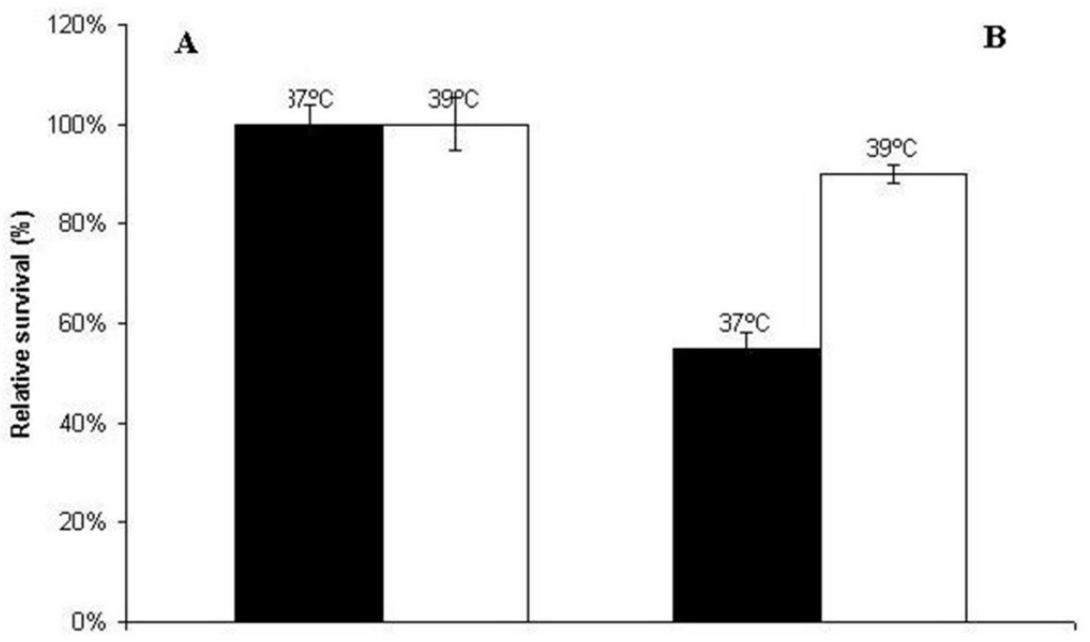

Figure 4: Survival of S. Abortusovis after pretreating the cells for $\mathbf{3 0}$ min with heat shock at $39^{\circ} \mathrm{C}$ and then exposed to antibiotic (nalidixic acid) stress.

A: Control cultures performed in the absence of stress; B: Cultures exposed to 45 $\mu \mathrm{g}$ nalidixic acid $\mathrm{ml}^{-1}$. Values represent percentages of cell survival (means of the three independent experiments) \pm the standard deviation (S.D.).

Excessive use of antibiotic for therapeutic reasons but also for growth promotion in farm animals selects for multiresistant bacterial strains. The occurrence and spread of multiresistant Salmonella strains was reported for many serovars and antibiotics (Esaki et al., 2004; Ngwai et al., 2005; Butt et al. 2006; Ercis et al., 2006; Karatzas et al., 2008; Strahilevitz et al., 2009; Gunell et al., 2009; Asai et al., 2010).

The mechanisms of antibiotic-resistance are different depending on the type of antimicrobial, and they can be represented by the drug inactivation or modification, target alteration or reduced accumulation associated with increased efflux or decreased permeability (Poole, 2002). The components of the antibiotic resistance mechanism are generally proteins that will be rapidly overexpressed when bacterial cells are damaged by antibiotics (Peleg et al., 2008; Cardoso et al., 2010).
Although further studies are needed to elucidate the role of molecular chaperones in the antibiotic stress response, the results obtained in this study showed that two of these proteins, DnaK and GroEL, are upregulated at the transcriptional and translational level by heat shock and different antibiotics (Table 2 and Figure 3). This major increase of molecular chaperones expression level could help to improve the ability of the S. Abortusovis to tolerate the stress caused by antibiotics.

Some studies have suggested a role of HSPs in bacterial stress response caused by antibiotics as fluoroquinolone, aminoglycosides, tetracyclines, beta-lactam antibiotics. The authors have found that DnaK, GroEL and Lon protease are some of stress proteins which are known to play a role in protein quality control that might contribute to antibiotic resistance because they are induced when the cells are subjected to this kind of stress (Kragol et al., 2001; Singh et al., 2001; Yamaguchi et al., 2003; Singh et al., 2007; Cardoso et al., 2010).

Our data represent a contribution to the understanding of the molecular chaperones role in antibiotic resistance machinery used by the bacterial cells to survive in the 
presence of various antibiotics that became ineffective in the treatment of infections. The multidrug-resistant strains of $\mathrm{S}$. Abortusovis indicate the necessity for the development of new antibiotics. DnaK and GroEL could be used as a target for these novel antimicrobials effective in the infection caused by S. Abortusovis.

The present study represents the first transcription and translation analysis of the molecular chaperones in a hostrestricted S. Abortusovis, regarding the response of this bacterium to heat shock and antibiotic stress conditions. Partially, these data could be considered preliminary and they will be mentioned in a future study.

\section{References}

1. Asai T., Sato C., Masani K., Usui M., Ozawa M., Ogino T., Aoki H., Sawada T., Izumiya H., Watanabe H. (2010). "Epidemiology of plasmid-mediated quinolone resistance in Salmonella enterica serovar typhimurium isolates from foodproducing animals in Japan", BioMed Central, Gut Pathogens, 2(7), 1-5.

2. Benndorf D., Loffhagen N., and Babel W. (1999). „Induction of heat shock proteins in response to primary alcohols in Acinetobacter Electrophoresis, 20, 781-789. calcoaceticus",

3. Benndorf D., Loffhagen N., and Babel W. (2001). „Protein synthesis patterns in Acinetobacter calcoaceticus induced by phenol and catechol show specificities of responses to chemostress", FEMS Microbiology Letters, 200, 247-252.

4. Beuzón C. R., Schiaffino A., Leori G., Cappuccinelli P., Rubino S. and Casadesús J. (1997). "Identification of Salmonella abortusovis by PCR amplification of a serovar-specific IS200 element", Applied Environmental Microbiology, 63(5), 20822085.

5. Bianchi, A.D. and Baneyx F. (1999). "Stress responses as a tool to detect and characterize the mechanism of action of antibacterial agents", Applied
Environmental Microbiology, 65, 50235027.

6. Blaszczak A., Zylicz M., Georgopoulos C., and Liberek K. (1995)."Both ambient temperature and the DnaK chaperone machine modulate the heat shock response in Escherichia coli by regulating the switch between $\sigma^{70}$ and $\sigma^{32}$ factors assembled with RNA polymerase", EMBO Journal, 14, 5085-5093.

7. Butt T., Khan M. Y., Ahmad R. N., Salman M., Afzal R. K. (2006). "Validity of nalidixic acid screening in Fluoroquinolone-resistant typhoid salmonellae", Journal of the College of Physicians and Surgeons Pakistan, 16(1), 31-34.

8. Cardoso K., Ferreira Gandra R., Wisniewski E. S., Aoki Osaku C., Kimiko Kadowaki M., Felipach-Neto V., Aby-Azar Haus L. F., and de Cássia Garcia Simão R. (2010). "DnaK and GroEL are induced in response to antibiotic and heat shock in Acinetobacter baumannii", Journal of Medical Microbiology, 59, 1061-1068.

9. Clinical and Laboratory Standards: Performance standards for antimicrobial disk and dilution susceptibility tests for bacteria isolated from animals. Clinical and Laboratory Standards Institute. (2008). Wayne, PA: CLSI.

10. Cudic M., Condie B. A., Weiner D. J., Lysenko E. S., Xiang Z. O., Insug O., Bulet P., and Otvos L. Jr. (2002). "Development of novel antibacterial peptides that kill resistant isolates", Peptides, 23, 2071-2083.

11. Ercis S., Erdem B., Hascelik G., Gür D. (2006). "Nalidixic acid resistance in Salmonella strains with decreased susceptibility to ciprofloxacin isolated from humans in Turkey", Japanese Journal of Infectious Diseases, 59(2), 117-119.

12. Esaki H., Chiu C-H, Kojima A., Ishihara K., Asai T., Tamura Y., and Takahashi T. (2004). "Comparison of fluoroquinolone resistance genes of Salmonella enterica serovar choleraesuis isolates in Japan and 
Taiwan", Japanese Journal of Infectious Diseases, 57, 287-288.

13. Gomes S. L., and Simão R. C. G. (2009). "Stress response: heat", Encyclopedia of Microbiology, 464-474.

14. Grimont P.A.D. and Weill F-X (2007). "Antigenic formulae of the Salmonella serovars", 9th edition, World Health Organization Collaborating Centre for Reference and Research on Salmonella, Institute Pasteur, Paris, France.

15. Gunell M., Webber M. A., Kotilainen P., Lilly A. J., Caddick J. M., Jalava J., Huovinen P., Siitonen A., Hakanen A. J., Piddock L. J. (2009). "Mechanisms of resistance in nontyphoidal Salmonella enterica strains exhibiting a nonclassical quinolone resistance phenotype", Antimicrobial Agents and Chemotherapy, 53(9), 38323836.

16. Jack E. J. (1968). "Salmonella abortion in sheep", Veterinary Annual, 12, 57-63.

17. Karatzas K. A., Randall L. P., Webber M., Piddock L. J., Humphrey T. J., Woodward M. J., and Coldham N. G. (2008). "Phenotypic and proteomic characterization of multiply antibiotic-resistant variants of Salmonella enterica serovar Typhimurium selected following exposure to disinfectants", Applied and Environmental Microbiology, 74(5), 1508-1516.

18. Kragol G., Lovas S., Varadi G., Condie B. A., Hoffmann R., and Otvos L. Jr. (2001). “ The antibacterial peptide pyrrhocoricin inhibits the ATPase actions of DnaK and prevents chaperone-assisted protein folding", Biochemistry, 40, 3016-3026.

19. Livak K. J. and Schmittgen T. D. (2001). "Analysis of relative gene expression data using real-time quantitative PCR and the 2(-Delta Delta C(T)) Method", 25 (4), 402408.

20. Mackey B. M., Derreck C. (1990). "Heat shock protein synthesis and thermotolerance in Salmonella typhimurium", Journal of Applied Bacteriology, 69(3), 373-383.
21. Morgan R. W., Christman M. F., Jacobson F. S., Storz G., and Ames B. N. (1986). "Hydrogen peroxide-inducible proteins in Salmonella typhimurium overlap with heat shock and other stress proteins", Proceedings of the National Academy of Sciences of the United States of America, 83, 8059-8063.

22. Ngwai Y., Ochi K., Ogawa Y., and Adachi Y. (2005). "Analysis of the protein profiles of the antibiotic-resistant Salmonella typhimurium definitive phage type (dt) 104", African Journal of Biotechnology, 4, 727-737.

23. Pardon P., Sanchis R., Marly J., Lantier F., Pépin M., Popoff M. (1988). "Ovine salmonellosis by Salmonella abortusovis" [in French], Annales de Recherches Véterinaires, 19(4), 221-235.

24. Peleg A. Y., Seifert H., and Peterson D. L. (2008). "Acinetobacter baumannii: emergence of a successful pathogen", Clinical Microbiology Reviews, 21, 538-582.

25. Poole K. (2002). "Mechanisms of bacterial biocide and antibiotic resistance", Journal of Applied Microbiology, 92, 55S$64 \mathrm{~S}$.

26. Prasad N. G., Dey S., Shakarad M., Joshi A. (2003) "The evolution of population stability as a by-product of life-history evolution", Proceedings of the Royal Society - biological sciences, 270(Suppl. 1) S84-S8.

27. Singh V. K., Jayaswal R. K., and Wilkinson B. J. (2001). "Cell wall-active antibiotic induced proteins of Staphylococcus aureus identified using a proteomic approach", FEMS Microbiology Letters, 199, 79-84.

28. Singh V. K., Utaida S., Jackson L. S., Jayaswal R. K., Wilkinson B. J., and Neal R. (2007). "Role for dnaK locus in tolerance of multiple stresses in Staphylococcus aureus", Microbiology, 153, 3162-3173

29. Strahilevitz J., Jacoby G. A., Hooper D. C., and Robicsek A. (2009). "Plasmidmediated quinolone resistance: a 
multifaceted threat", Clinical Microbiology Reviews, 22(4), 664-689.

30. Susin M. F., Baldini R. L., Gueiros-Filho F., and Gomes S. L. (2006). "GroES/GroEL and DnaK/DnaJ Have Distinct Roles in Stress Responses and during Cell Cycle Progression in Caulobacter crescentus", Journal of Bacteriology, 188(23), 80448053.

31. Uzzau S., Brown D. J., Wallis T., Rubino S., Leori G., Bernard S., Casadesús J., Platt D. J., Olsen J. E. (2000). "Host adapted serotypes Salmonella enterica", Epidemiology and Infection, 125, 229-255.
32. Vanghele L. M., Ionescu M., Coste H., and Ganea E. (2013). "Induction of DnaK and GroEL in Brucella ovis Under Various Stress Conditions", International Journal of Veterinary Medicine: Research \& Reports, 2013, 1-11.

33. Yamaguchi Y., Tomoyasu T., Takaya A., Morioka M., and Yamamoto T. (2003). "Effects of disruption of heat shock genes on susceptibility of Escherichia coli to fluoroquinolones", BMC Microbiology, 3, 1623. 